\title{
E3 Ubiquitin-Protein Ligase HERC2
}

National Cancer Institute

\section{Source}

National Cancer Institute. E3 Ubiquitin-Protein Ligase HERC2. NCI Thesaurus. Code C117251.

E3 ubiquitin-protein ligase HERC2 (4834 aa, 527 kDa) is encoded by the human HERC2 gene. This protein is involved in ubiquitin-dependent retention of DNA repair proteins. 